\title{
Clinical Correlation of Maximum Standardised Uptake Value (SUVmax) in Positron Emission Tomography- Computed Tomography (PET-CT) with Initial Disease Staging of Carcinoma Breast- An Observational Study at Indian Tertiary Care Centre
}

\author{
Gokulakrishnan Periakaruppan¹, Bharathi Vivekanadan Sambasivam², Arunan Murali ${ }^{3}$
}

${ }^{1}$ Department of Radiology, Sri Ramachandra Institute of Higher Education and Research, Chennai, Tamilnadu, India. ${ }^{2}$ Department of Radiology, Sri Ramachandra Institute of Higher Education and Research, Chennai, Tamilnadu, India.

${ }^{3}$ Department of Radiology, Sri Ramachandra Institute of Higher Education and Research, Chennai, Tamilnadu, India.

\section{ABSTRACT}

\section{BACKGROUND}

The most common primary malignant disease in the world amongst women is carcinoma breast. Metabolic activity of a lesion (SUVmax) has strong clinical correlation with various prognostic factors according to numerous literature reviews. The main aim of this study is to assess the correlation between maximum Standardized Uptake Value [SUVmax] and initial disease staging (as per AJCC TNM$8^{\text {th }}$ edition). We wanted to study the correlation between metabolic activity [SUVmax] of the primary tumour in breast carcinoma, and size of the primary, nodal and distant metastatic status.

\section{METHODS}

This is an observational study conducted over a period of two years in breast cancer patients $[\mathrm{n}=139]$ undergoing PET-CT as a part of initial staging. PET-CT was done using Siemens Horizon True-V PET according to institutional protocols.

\section{RESULTS}

Significantly higher SUVmax values were observed in tumours with larger size [ $>2$ $\mathrm{cms}$ ]. However, there was no significant correlation between SUVmax of primary tumours and the status of axillary nodal involvement and distant metastases $[\mathrm{p}=$ 0.125 and 0.847 respectively].

\section{CONCLUSIONS}

Metabolic activity of primary breast cancer has strong clinical correlation with size; however, there is no such correlation found in nodal and metastatic spread of the disease.

\section{KEY WORDS}

Carcinoma Breast, PET-CT, Tumour Size, Axillary Nodes, Distant Metastasis
Corresponding Author:

Dr. Gokulakrishnan Periakaruppan, Department of Radiology,

Sri Ramachandra Institute of Higher Education and Research, Chennai,

Tamilnadu, India.

E-mail: raygokul@gmail.com

DOI: $10.14260 / \mathrm{jemds} / 2020 / 311$

Financial or Other Competing Interests: None.

How to Cite This Article:

Periakaruppan G, Sambasivam BV, Murali A. Clinical correlation of maximum standardised uptake value (SUVmax) in positron emission tomography- computed tomography (PET-CT) with initial disease staging of carcinoma breast- an observational study at Indian tertiary care centre. J. Evolution Med. Dent. Sci. 2020;9(17):1427-1430, DOI: $10.14260 /$ jemds $/ 2020 / 311$

Submission 16-03-2020, Peer Review 20-04-2020 Acceptance 22-04-2020, Published 27-04-2020.
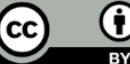


\section{BACKGROUND}

Various diagnostic and screening modalities and protocols are being formulated and constantly revised to help in the evaluation of breast carcinoma to help in the early diagnosis and accurate staging of the tumour, so as to initiate appropriate therapy at the earliest. Before initiating therapy, it is highly recommended to perform a staging/metastatic workup, ${ }^{[1-3]}$ and obtain the histopathological characteristics of the tumour.[1-3] Multiple new treatment modalities are available, which are tailored to the grade and stage of the tumour. ${ }^{[2-4]}$ Newer drugs targeted at the molecular level are also available which are selected according to the hormonal receptor expression by the tumour cells.[5] The prognosis of the patients significantly improves when the pre-treatment staging is accurate and the stage-appropriate therapy guidelines are followed.[6]

Previously, metastatic workup used to consist of a chest X-ray, ultrasonography screening of abdomen and Bone scintigraphy. As these consist of multiple individual tests and diagnostic yield of the above modalities in detecting early metastatic disease is quite low, the use of PET-CT as a single modality for screening the whole body for metastases is quite beneficial,[7] as the patient compliance and diagnostic sensitivity is better as compared to conventional modalities.[8-12]

Previous imaging modalities like USG, Mammogram and CT/MRI are designed to provide anatomical information with good resolution, but they lack any functional information. Nuclear scintigraphy, PET and other nuclear imaging studies are capable of providing information about the metabolic activity of the tumour under evaluation. However, their major drawbacks include poor anatomical localisation and low spatial resolution. With the advent of PET-CT fusion imaging those drawbacks has been rectified and the resultant imaging data provides combined anatomical and functional information with excellent spatial resolution and precise anatomical localisation.

PET-CT is performed by injecting the patient with radioactive isotope [18F- Fluro Deoxy Glucose in our case] and scanning the patient. The radioisotope accumulates within the tissues with high metabolic activity i.e., tumour cells and these tissues show up as areas of increased uptake [hot-spots]. The PET metabolic data is fused with CT anatomical/morphologic data, which help us localise the tumour better.[13] PET-CT also plays a significant role in detecting and localising metastases.[13]

\section{METHODS}

An observational study was conducted in radiology department of Sri Ramachandra Institute of Higher Education and Research from November 2017 to October 2019 in 139 consecutive eligible patients who were referred for whole body PET-CT for diagnosis and staging of Primary breast carcinoma. Institutional Ethics committee approval was obtained for the study. Informed consent was obtained from all the patients.

\section{Inclusion Criteria}

- Patients undergoing primary staging evaluation for breast carcinoma.

- Patients with complete histopathological data. i.e., histological type, Nottingham grading and immunehistochemistry panel including Oestrogen receptor status [ER], Progesterone receptor status [PR], HER-2 receptor status $[\mathrm{HR}]$ and ki-67 labelling index.

\section{Exclusion Criteria}

- Patients who have general contraindications for undergoing PET-CT such as pregnancy and breastfeeding.

- Patients who have undergone any form of treatment before PET-CT such as chemotherapy, radiotherapy or any form of allopathic/homeopathic medications targeted for breast carcinoma.

- Patients without the required histopathological data/ immunohistochemistry markers.

\section{Materials and Methods}

- Equipment used: Siemens Biograph Horizon TRUE V PET/CT.

- Software: syngo.via version VB30A.

- Radiotracer Used: 18F-Flurodeoxyglucose [Dose: 5-10 $\mathrm{mCi}]$.

- Contrast Media Used: Iohexol [Trade name - Omnipaque $350 \mathrm{mg} / \mathrm{dL}$ ] [dose $-1.5 \mathrm{mg} / \mathrm{kg}$ ]

\section{Statistical Analysis}

Statistical analysis is done by independent sample-t test with significance set at $\mathrm{p}<0.05$.

\section{RESULTS}

\section{Difference in SUVmax Value of Primary Tumour in} Relation to Tumour Size

In keeping with the previous literature, we divided the patients into two groups, with the first group [n $=33]$ consisting of patients with primary tumours $\leq 2 \mathrm{~cm}$ in size [greatest dimension] and the second group [ $\mathrm{n}=106]$ consisting of patients with tumours $>2 \mathrm{~cm}$. The mean $\pm \mathrm{SD}$ value of SUVmax of primary tumours $\leq 2 \mathrm{~cm}$ was $6.0 \pm 4.6$ and that of tumours $>2 \mathrm{~cm}$ was $10.83 \pm 8.46$. The difference between the two groups was found to be statistically significant $[\mathrm{p}=0.002]$ as shown in table 1 .

\begin{tabular}{|c|c|c|c|}
\hline & No. of Cases & Mean SUVmax & SD \\
\hline Size $\leq 2 \mathrm{~cm}$ & 33 & 6.0 & 4.6 \\
\hline Size $>2 \mathrm{~cm}$ & 106 & 10.83 & 8.46 \\
\hline \multicolumn{2}{|c|}{ Independent sample t-test } & Significance $(p)$ & 0.002 \\
\hline
\end{tabular}

Correlation of SUVmax Value of Primary Tumour with Axillary Nodal Involvement

In our study of 139 patients, 100 patients had axillary nodal involvement at the time of initial scan and 39 patients did not 
have axillary nodal involvement. The mean \pm SD of SUVmax values of cases with axillary nodal involvement and without axillary nodal involvement were $10.37 \pm 7.73$ and $7.93 \pm 8.5$ respectively. Even though the mean SUVmax of tumours with axillary nodal involvement was higher [ 10.37 vs. 7.93 ], the difference between the groups was not statistically significant [p $=0.125]$ as shown in table 2 .

\begin{tabular}{|c|c|c|c|c|}
\hline & $\mathbf{n}$ & Frequency & Mean SUVmax & SD \\
\hline No axillary nodal spread & 39 & $28.1 \%$ & 7.93 & 8.5 \\
\hline Axillary node spread + & 100 & $71.9 \%$ & 10.37 & 7.73 \\
\hline & & $\mathrm{F}$ & \multicolumn{2}{|c|}{ Significance $(p)$} \\
\hline \multicolumn{2}{|c|}{ Independent samples t-test } & 0.156 & \multicolumn{2}{|c|}{0.125} \\
\hline $\begin{array}{r}\text { Table 2. Ind } \\
\text { SUVmax Value }\end{array}$ & enc & $\begin{array}{l}\text { ample T-T } \\
\text { us of Axilla }\end{array}$ & $\begin{array}{l}\text { for Correlating } \\
\text { odal Involveme }\end{array}$ & \\
\hline
\end{tabular}

\section{Correlation of SUVmax Value of Primary Tumour with} Presence of Distant Metastases

The mean \pm SD of SUVmax value of cases with distant metastases $[n=41]$ at the time of initial scan was $9.86 \pm 5.77$. And the mean \pm SD of SUVmax value of cases without distant metastases $[\mathrm{n}=98]$ at the time of initial scan was $9.61 \pm 8.71$. There was no statistically significant relationship between the two groups [ $p=0.847]$ as shown in table 3 .

\begin{tabular}{|ccccc|}
\hline & n & Frequency & Mean SUVmax & SD \\
No distant metastases & 98 & $70.5 \%$ & 9.61 & 8.79 \\
Distant metastases + & 41 & $29.5 \%$ & 9.86 & 5.77 \\
\multicolumn{3}{c}{ Independent samples t-test } & $\mathrm{F}$ & \multicolumn{2}{c|}{ Significance (p) } \\
\hline \multicolumn{3}{|c|}{ Table 3. Independent Sample T-Test for Correlating } \\
SUVmax Values with Presence or Absence of Distant Metastases \\
\hline
\end{tabular}

\section{DISCUSSION}

In our study the mean \pm SD value of SUVmax of the tumours $\leq$ $2 \mathrm{~cm}$ in size was $6.0 \pm 4.6$ and the mean \pm SD value of SUVmax of the tumours $>2 \mathrm{~cm}$ was $10.83 \pm 8.46$. The tumours $>2 \mathrm{~cm}$ had statistically significant $[p=0.002]$ higher SUVmax values. Our findings are comparable with Ito et al.,[14] who also observed that tumours $\leq 2 \mathrm{~cm}$ had significantly lesser SUVmax than tumours $>2 \mathrm{~cm}$ [SUVmax 2.2 vs 3.1 respectively]. In the same note, Higuchi et al.,[15] also found that tumours larger than $2 \mathrm{~cm}$ had statistically higher SUVmax values $(\mathrm{p}=0.011)$. They also defined tumour size as an independent prognostic factor for survival in patients with breast cancer. Arslan et al.,[16] also obtained similar results in their study where tumours smaller than $2 \mathrm{~cm}$ had significantly lower SUVmax values than larger tumours [mean SUVmax of 8.1 vs $13.0, \mathrm{p}<0.001$ ].

One of the most important advantages of PET-CT in the evaluation of breast carcinoma is its sensitivity and accuracy in detecting axillary, non-axillary lymph nodal spread and distant metastases.[17] In our study, no relationship was found between the SUVmax values in cases with distant metastases $[9.86 \pm 5.77]$ and cases without distant metastases [9.61 \pm 8.79], with p-value of 0.847 . Also, the FDG uptake of primary tumours was higher in cases with axillary nodal involvement [mean \pm SD value of SUVmax $=10.37 \pm 7.73$ in axillary node positive cases and $7.93 \pm 8.5$ in axillary node negative cases]. Even though the cases with axillary nodal involvement had higher SUVmax values, the difference was not statistically significant [0.125]. Our results could not support the findings in few previous studies by Basu et al.,[18] and Koolen at el.,[19] who observed that the SUVmax values of the primary tumours were higher in patients with axillary and distant metastases. Abubakar et al.,[20] found that tumours with positive axillary nodal spread has higher SUVmax values as compared to the node negative group (mean SUVmax of 12.5 vs. 7.8; $\mathrm{p}<0.0001$ ). But this study did not find any significant relation between the SUVmax of the tumour and the presence of distant metastases as found in our study.

\section{CONCLUSIONS}

SUVmax of tumours $>2 \mathrm{cms}$ size was significantly higher than that of tumours $\leq 2 \mathrm{cms}[\mathrm{p}=0.002]$. No significant correlation was observed between SUVmax of primary tumours and the status of axillary nodal involvement and distant metastases [p $=0.125$ and 0.847 respectively].

\section{Data Availability}

The data can be made available to the readers upon request to corresponding author of this article.

\section{REFERENCES}

[1] Somashekhar SP, Agarwal G, Deo SVS, et al. Indian solutions for Indian problems-Association of Breast Surgeons of India (ABSI) practical consensus statement, recommendations and guidelines for the treatment of breast cancer in India. Indian J Surg 2017;79(4):275-85.

[2] Cardoso F, Kyriakides S, Ohno S, et al. Early breast cancer: ESMO clinical practice guidelines for diagnosis, treatment and follow-up. Ann Oncol 2019;30(8):1194220.

[3] Gradishar WJ, Anderson BO, Balassanian R, et al. Breast Cancer, Version 4.2017, NCCN clinical practice guidelines in oncology. J Natl Compr Canc Netw 2018;16(3):310-20.

[4] Liedtke C, Jackisch C, Thill $M$, et al. AGO Recommendations for the diagnosis and treatment of patients with early breast cancer: update 2018. Breast Care (Basel) 2018;13(3):196-208.

[5] Andre F, Ismaila N, Henry NL, et al. Use of biomarkers to guide decisions on adjuvant systemic therapy for women with early-stage invasive breast cancer: ASCO clinical practice guideline update-integration of results from TAILORx. J Clin Oncol 2019;37(22):1956-64.

[6] Varga D, Wischnewsky M, Atassi Z, et al. Does guidelineadherent therapy improve the outcome for early-onset breast cancer patients? Oncology 2010;78(3-4):189-95.

[7] Eubank WB, Mankoff DA, Vesselle HJ, et al. Detection of locoregional and distant recurrences in breast cancer patients by using FDG PET. Radiographics 2002;22(1):517.

[8] Morris PG, Lynch C, Feeney JN, et al. Integrated positron emission tomography/computed tomography may render bone scintigraphy unnecessary to investigate suspected metastatic breast cancer. J Clin Oncol 2010;28(19):3154-9. 
[9] Lim HS, Yoon W, Chung TW, et al. FDG PET/CT for the detection and evaluation of breast diseases: usefulness and limitations. Radiographics 2007;27(Suppl 1):S197213.

[10] Groheux D, Giacchetti S, Espié M, et al. The yield of 18FFDG PET/CT in patients with clinical stage IIA, IIB or IIIA breast cancer: a prospective study. J Nucl Med 2011;52(10):1526-34.

[11] Liu Y. Role of FDG PET-CT in evaluation of locoregional nodal disease for initial staging of breast cancer. World J Clin Oncol 2014;5(5):982-9.

[12] Garg PK, Deo SVS, Kumar R. Role of positron emission tomography-computed tomography in locally advanced breast cancer. Indian J Surg Oncol 2015;6(4):420-6.

[13] Yang SK, Cho N, Moon WK. The role of PET/CT for evaluating breast cancer. Korean J Radiol 2007;8(5):42937.

[14] Ito M, Shien T, Kaji M, et al. Correlation between 18Ffluorodeoxyglucose positron emission tomography/computed tomography and clinicopathological features in invasive ductal carcinoma of the breast. Acta Med Okayama 2015;69(6):333-8.

[15] Higuchi T, Nishimukai A, Ozawa H, et al. Prognostic significance of preoperative 18F-FDG PET/CT for breast cancer subtypes. Breast Edinb Scotl 2016;30:5-12.

[16] Yildirim N, Simsek M, Aldemir MN, et al. Relationship between 18-FDG-PET/CT and clinicopathological features and pathological responses in patients with locally advanced breast cancers. Eurasian J Med 2019;51(2):154-9.

[17] Groheux D, Hindié E. Is (18) FDG uptake useful to decide on chemotherapy in ER+/HER2- breast cancer? Eur J Nucl Med Mol Imaging 2016;43(9):1571-3.

[18] Basu S, Mavi A, Cermik T, et al. Implications of standardized uptake value measurements of the primary lesions in proven cases of breast carcinoma with different degree of disease burden at diagnosis: does 2deoxy-2-[F-18]fluoro-D-glucose-positron emission tomography predict tumour biology? Mol Imaging Biol 2008;10(1):62-6.

[19] Koolen BB, Peeters MJTFDV, Wesseling J, et al. Association of primary tumour FDG uptake with clinical, histopathological and molecular characteristics in breast cancer patients scheduled for neoadjuvant chemotherapy. Eur J Nucl Med Mol Imaging 2012;39(12):1830-8.

[20] Abubakar Z, Akepati NKR, Bikkina P. Correlation of maximum standardized uptake values in $18 \mathrm{~F}$ Fluorodeoxyglucose positron emission tomographycomputed tomography scan with immunohistochemistry and other prognostic factors in breast cancer. Indian J Nucl 2019;34(1):10-13. 